\title{
Pacientes referidos al Centro Estatal de Vasectomías, factores para Ia aceptación o rechazo del método
}

\author{
García-Franco María Evangelina*, Hernández-Flores Olga Lydia*, Góngora-Ortega Javier**
}

\section{Resumen}

- Objetivo: Identificar los factores socio-culturales que intervie-

- nen en la aceptación y el rechazo de vasectomía en hombres

- que utilizan los servicios de salud de un medio urbano en la

- ciudad de Aguascalientes. Metodología: Se realizó un estudio

- observacional, transversal, descriptivo y comparativo, incluyendo

- 48 hombres que aceptaron realizarse la vasectomía y 48 que

- la rechazaron, muestreados aleatoriamente del Centro Estatal

- de Vasectomía de la Secretaría de Salud. Se midieron variables

- socio-culturales, conocimientos en vasectomía y planificación

- familiar (PF) y se compararon ambos grupos. Resultados: Los

- hombres que rechazaron la vasectomía tenían 70.4 \% con ni-

- vel escolar de preparatoria o superior mientras los que la acep-

- taron tenían 87.2\% ( $p=0.049)$. En el nivel de conocimientos,

- los que rechazaron la vasectomía obtuvieron una calificación

- promedio de $17.0 \pm 3.6$ puntos y quienes la aceptaron 18.7

- \pm 3.2 puntos ( $p=0.017$ ); cuando el paciente fue informado

- sobre la vasectomía por amistades, el nivel de conocimientos

- de quienes la rechazaron fue de $14.5 \pm 4$.I puntos, mientras

- los que la aceptaron fueron $19.5 \pm 3.2$ puntos ( $p=0.002$ ).

- Conclusiones: Incrementar el conocimiento sobre este método

- y considerar la importancia de las amistades, puede mejorar

- la aceptación de los hombres. LUX MÉ́DICA AÑO 8, NÚMERO 25,

- SEPTIEMBRE-DICIEMBRE 2013,PP 11-22

-

Palabras clave: Vasectomía, conocimientos, factores socioculturales.

\section{Abstract}

Objective: To identify socio-cultural factors involved in the acceptance and rejection of vasectomy in men who used health services in an urban media in the City of Aguascalientes. Methodology: An observational, cross sectional, descriptive and comparative study was conducted including 48 men that accepted to performed vasectomy and 48 who rejected it. Randomly sampled from the State Center of vasectomy from the Ministry of Health, sociocultural variables, knowledge about vasectomy and both groups were compared.

Results: Men who rejected vasectomy had $70.4 \%$ with grade level high school or higher, while those who accepted it had $87.2 \%(p=0.049)$. In the level of knowledge about vasectomy, which rejected the vasectomy obtained a mean score 17.0 \pm 3.6 points and those who rejected it $18.7 \pm 3.2$ points ( $p$ $=0.017)$; when the patient was informed about vasectomy by friendships, the level of knowledge of who rejected it was $14.5 \pm 4.1$ points, while those who accepted it were $19.5 \pm$ 3.2 points ( $p=0.002)$. Conclusions: Increase knowledge about this method and consider the importance of friendships can improve the acceptance of men. LUXMÉDICA AÑ08, NÚMERO25, SEPTIEMBRE-DICIEMBRE 2013, PP 11-22

Key words: Vasectomy, knowledge, socio-cultural factors

Licenciadas en Enfermería de la Secretaría de Salud del Estado de Aguascalientes

** Maestro en Ciencias de la Secretaría de Salud del Estado de Aguascalientes

Fecha de recibido: 17 de septiembre 2013

Fecha de aceptación: 9 de octubre 2013

Correspondencia: MC Javier Góngora Ortega. Calle Margil de Jesús \#1501 Fraccionamiento Arboledas CP 20020 Aguascalientes, Ags. México. Teléfono 01 (449) 9107900 ext 7266 Fax: 01449910798044919621 64, Dirección electrónica: javiergongora@hotmail.com 


\section{Introducción}

Los servicios de Planificación Familiar (PF) en México cuentan con métodos anticonceptivos temporales y permanentes, dentro de estos últimos se encuentran la oclusión tubárica bilateral y la vasectomía. ${ }^{1}$

La vasectomía es un método de control de la fecundidad masculina que inició en 1930 con Sharp, pero es en 1963 que Poffenberger publica resultados excelentes de esterilización en 2,000 casos intervenidos de 1955 a $1961 .^{2}$ El término vasectomía proviene del latín vas, conducto, y del griego ektome, que significa escisión. Consiste en el corte de los conductos deferentes para bloquear el paso de los espermatozoides con la finalidad de impedir que se incorporen al semen y salgan del organismo del varón por medio de la eyaculación. Tiene la ventaja de tener un menor índice de morbilidad y un menor costo que la esterilización femenina. ${ }^{3-5}$ En el centro de cirugía ambulatoria del Instituto de Seguridad y Servicios Sociales de los Trabajadores del Estado (ISSSTE) en México, el $93 \%$ de los hombres recomendaron la vasectomía sin bisturí por ser práctica, de bajo riesgo, segura, que elimina las preocupaciones de un embarazo no deseado, permitiendo que los hombres tomen una parte más activa en el control de la natalidad. ${ }^{6}$

A pesar de los beneficios evidenciados por los hombres que optan por la vasectomía, García y cols. ${ }^{7}$ encontraron que en los varones que la rechazan tienen franco temor al desempeño sexual posterior al procedimiento (disminución de la libido, disfunción eréctil, debilidad física) y enmascaran el temor argumentando que la anticoncepción es responsabilidad de la mujer. La salud sexual y reproductiva es uno de los ámbitos en los que se expresan con mayor fuerza las desigualdades e inequidades de género. ${ }^{8,9}$ Por otra parte Quiroz ${ }^{10}$ y cols, determinaron que tener una actitud positiva y un mayor nivel de información sobre métodos anticonceptivos por parte de los hombres, se relaciona con una mayor aceptación y uso de los mismos. Algunos expertos consideran que el poco involucramiento de los hombres para el control definitivo de la fertilidad se debe a creencias y actitudes negativas sobre la vasectomía; otros creen que es la combinación de fenómenos enraizados en la cultura, como el machismo y la influencia religiosa. 6,11,12

En un estudio realizado en hombres jóvenes de la Ciudad de México, se encontró que reconocen la importancia de los anticonceptivos; sin embargo traen consigo un pensamiento propio del patriarcado por las creencias que se sustentan en el placer sexual y la virilidad, donde éstos se sobreponen al uso de anticonceptivos. ${ }^{13}$ La vasectomía podría ser observada como el riesgo de una pérdida de la virilidad más que como un método de PF confiable.

Como se puede observar existen diversos factores que incrementan o diminuyen la posibilidad de que un hombre opte por la vasectomía como método de $\mathrm{PF}$, que pueden ser diferentes en cada región, dependiendo de las características socioculturales de 
la población. ${ }^{14}$ La finalidad del presente estudio es conocer los factores socioculturales que pueden estar condicionando el aceptar o rechazar la vasectomía en hombres de un medio urbano, que fueron canalizados al módulo estatal de vasectomías de la Secretaría de Salud de Aguascalientes, México.

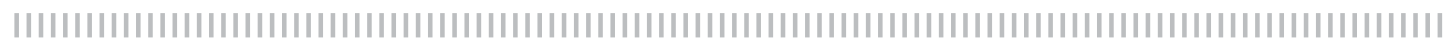

\section{Material y métodos}

Se realizó un estudio observacional, transversal, descriptivo, prospectivo y comparativo. Se incluyeron pacientes referidos por los centros de salud, al Módulo de Vasectomía de la Secretaría de Salud del Estado de Aguascalientes, un universo de 258 pacientes referidos, 171 fueron sometidos a la vasectomía y 87 no. Se realizó un muestreo probabilístico simple al interior de cada grupo, tomando en consideración una muestra de 48 pacientes por grupo, el tamaño se obtuvo mediante la fórmula de comparación de proporciones del programa Epi-info Ver 6.04 tomando en cuenta la variable de nivel escolar como la de mayor relevancia, con el $70 \%$ en el nivel de bachillerato o mas para los que fueron opera- dos y el $30 \%$ para los que no se operaron. Mediante visita domiciliaria se procedió a aplicar un instrumento creado ex profeso, revisado y validado en contenido por cuatro expertos en el tema (un maestro en ciencias, el responsable del programa de PF de la Secretaría de Salud a nivel estatal, la encargada del módulo de vasectomía y un experto en sexología), piloteado en diez hombres que se realizaron la vasectomía y diez que la rechazaron, la versión final consta de 45 ítems en 2 dominios: variables socioculturales y conocimientos sobre PF y vasectomía, el instrumento obtuvo una alfa de Cronbach de 0.69. Se utilizó la prueba $\mathrm{X}^{2}$ y $\mathrm{t}$ de student, para la comparación de grupos en el programa SPSS ver 17.0.

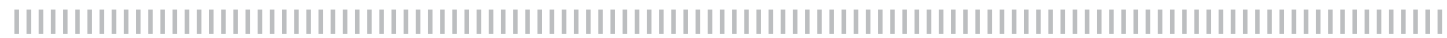

\section{Resultados}

Se entrevistaron 47 pacientes que aceptaron la vasectomía y 45 que no aceptaron, la edad promedio fue de $38.2 \pm$ 9.6 años, el principal estado civil, casado $(67.4 \%)$, seguido de unión libre $(14.1 \%)$. El nivel escolar que predominó fue técnico $(28.3 \%)$, seguido de licenciatura $(27.2 \%)$. Las dos frecuencias mas altas en el tipo de ocupación fueron empleado profesionista $(43.5 \%)$ y empleado no profesionista (41.3\%).

De los principales motivos para realizarse el procedimiento fueron: paridad satisfecha en un $50 \%$, obtener una mejor calidad de vida en un $20.3 \%$, por ser una cirugía de bajo riesgo y rápida en un $9.2 \%$. Los principales motivos de rechazo fueron: decidir tener más hijos (31.2\%), temor a quedarse impotentes (10.4\%) y haber elegido otro método anticonceptivo con el $10.4 \%$.

\section{Comparación entre grupos}

Al comparar la edad de los hombres, la edad de la pareja, número de hijos y años de matrimonio fueron similares $(p>0.05)$ en ambos grupos (aceptación y rechazo), resultando mayor la edad de los hombres que la de sus parejas (Tabla 1). Tampoco existieron diferencias significativas entre ambos grupos al comparar su estado civil, ocupación del paciente y ocupación de su pareja. 


\section{Tabla I}

Edad del hombre, de la pareja, años de matrimonio y número de hijos comparados por grupo de estudio. * $t$ de student.

\begin{tabular}{|lccc|}
\hline & $\begin{array}{c}\text { Rechazaron } \\
\text { (Prom } \pm \text { desv st) }\end{array}$ & $\begin{array}{c}\text { Aceptaron } \\
\text { (Prom } \pm \text { desv st) }\end{array}$ & Valor p $^{*}$ \\
\hline Edad del hombre & $39.0 \pm 9.2$ & $37.1 \pm 10.0$ & 0.44 \\
\hline Edad de la pareja & $34.3 \pm 8.6$ & $34.1 \pm 8.6$ & 0.92 \\
\hline Años de matrimonio & $12.1 \pm 7.6$ & $12.1 \pm 7.6$ & 0.98 \\
\hline Número de hijos & $2.6 \pm 1.3$ & $2.3 \pm 0.8$ & 0.12 \\
\hline
\end{tabular}

Por otra parte se encontró que los que rechazaron la vasectomía presentaron 70.4 $\%$ con un nivel de preparatoria o superior mientras que el porcentaje entre los que la aceptaron fue de un $87.2 \%$, con una diferencia estadísticamente significativa $\left(X^{2} p=\right.$ 0.049). Respecto a la religión, se observó que quienes rechazaron la vasectomía profesaban otras religiones aparte de la católica y la cristiana; mientras que los que la aceptaron, solo profesaban las religiones antes mencionadas, con una diferencia significativa $(p=0.049)$ (Tabla 2$)$.

\section{Tabla 2}

Religión que profesaban los pacientes de acuerdo al grupo de estudio.* 2 y 1 pacientes respectivamente no contestaron. ${ }^{* *} \mathrm{Ji}$ cuadrada. $\mathrm{P}=0.049$

\begin{tabular}{lcc}
\hline & Rechazaron & Aceptaron \\
& $\mathrm{n} \%$ & $\mathrm{n} \%$ \\
\hline Católica & 34 & 39 \\
& $79.1 \%$ & $84.7 \%$ \\
\hline Cristiana & 3 & 7 \\
& $7.0 \%$ & $15.2 \%$ \\
\hline Otra & 5 & 0 \\
& $11.6 \%$ & $0 \%$ \\
\hline Agnóstico & 1 & 0 \\
& $2.3 \%$ & $0 \%$ \\
\hline Total & $43^{*}$ & $46^{*}$ \\
& $100 \%$ & $100 \%$ \\
\hline
\end{tabular}

Al correlacionar el nivel de conocimientos sobre la vasectomía, con la aceptación o rechazo de la misma, encontramos que quienes la rechazaron obtuvieron una cali- ficación promedio de $17.0 \pm 3.6$ puntos y los que aceptaron $18.7 \pm 3.2$ puntos con una $p=0.017$ (t de student, Gráfica 1). 


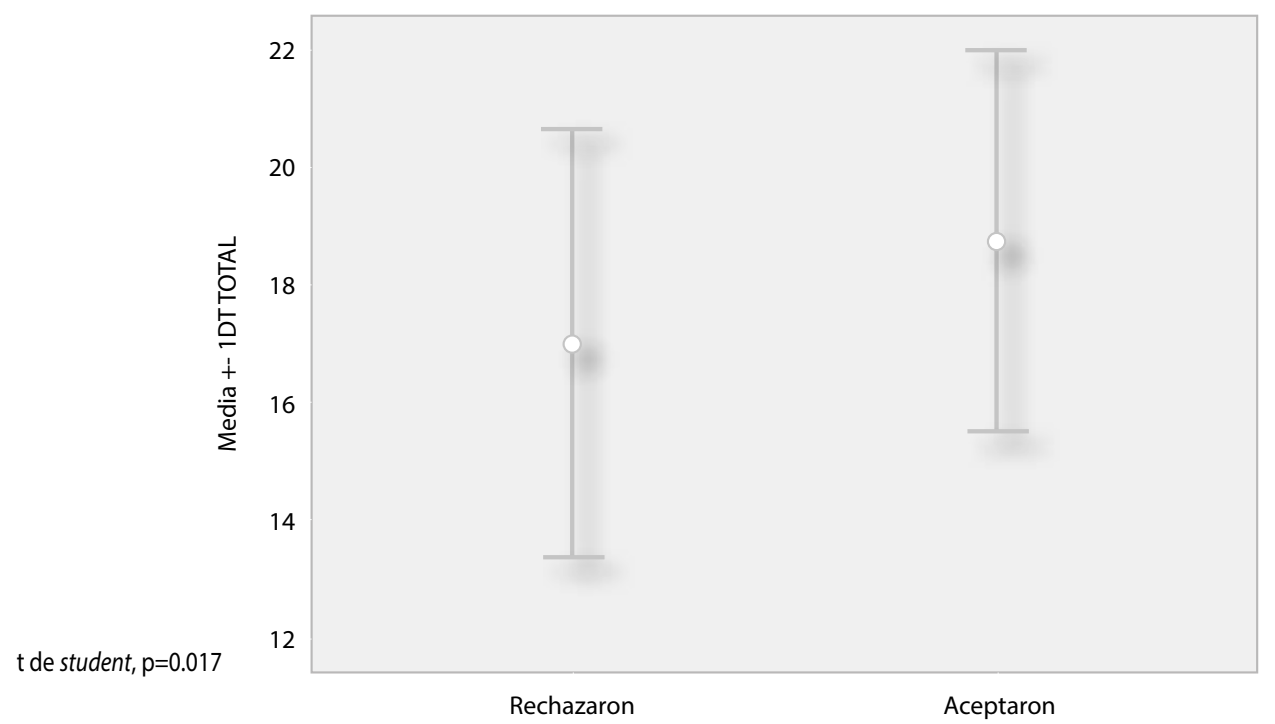

Gráfica 1. Nivel de conocimientos entre los que rechazaron y aceptaron la vasectomía.

\section{Análisis en sub grupos según de quien recibió información}

Se realizó un análisis considerando subgrupos, dependiendo por quién fue proporcionada la información: familiares, amistades, profesionales de la salud o medios masivos. Cuando la información fue proporcionada por amistades, el nivel de conocimientos del grupo que rechazó la vasectomía fue de $14.5( \pm 4.1$ puntos) mientras los que la aceptaron fueron 19.5 ( \pm 3.2 puntos), siendo el nivel más bajo y más alto respectivamente y con una diferencia estadísticamente significativa (prueba $t p=0.005)$; cuando la información fue proporcionada por familiares, personal de salud o medios masivos, no existieron diferencias en el nivel de conocimientos entre los que rechazaron o aceptaron la vasectomía (Gráfica 2).

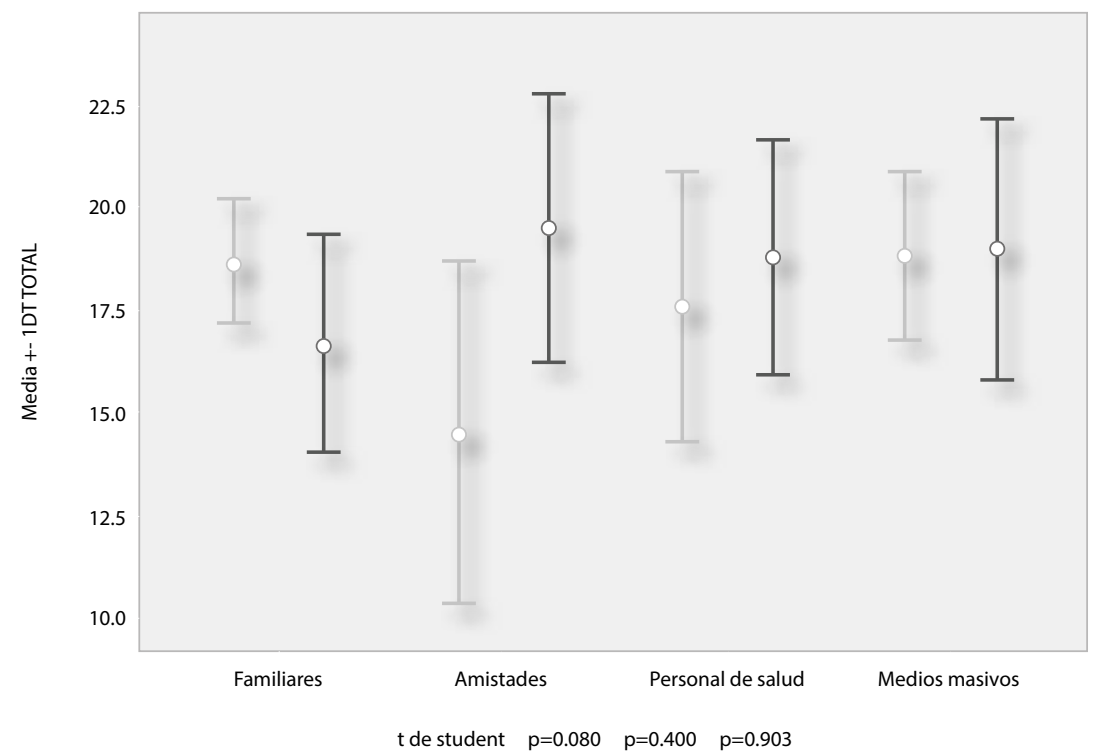

Gráfica 2. Nivel de conocimientos entre los que rechazaron y aceptaron la vasectomía, por quién dio la información. 


\section{Discusión}

Es Importante partir de las características de la población estudiada, el estudio se realizó en personas que habían sido referidas al centro estatal de vasectomía por algún centro de salud; por lo tanto eran hombres que ya tenían una información.

El nivel escolar es un factor importante para aceptar o rechazar la vasectomía, el resultado es similar al reportado por LaraRicalde $^{15}$ y cols, quien encontró que los pacientes que se realizan la vasectomía tienen predominantemente un nivel escolar de preparatoria o mayor.

Respecto de la variable edad podemos decir que no existen diferencias entre los que aceptaron y la rechazaron, contra lo que se podría esperar que la variable edad no siempre es predictora de diferencias en las opiniones y actitudes de los varones respecto de la aceptación o no de la vasectomía; resultados que concuerdan por el estudio realizado por Edith Pantelides. ${ }^{16}$

Los principales motivos que se observaron para aceptar realizarse la vasectomía no difieren de lo reportado por otros estudios, ${ }^{3}$ pero por otra parte el motivo del rechazo como el desear tener más hijos pareciera ser el ocultamiento de otros motivos, como pudiera ser un deficiente desempeño sexual posterior, en varones que argumentan de primera instancia que la anticoncepción es responsabilidad de la mujer. $^{7}$

Encontramos que a mayor nivel de conocimientos sobre PF y la vasectomía, mayor aceptación de la vasectomía por parte de los hombres, los resultados son acordes con los de Quiroz, ${ }^{10}$ el cual refiere que a un mayor nivel de información sobre métodos anticonceptivos por parte de los varones se relaciona con una mayor aceptación y uso de los mismos

Esto se refleja también con el hecho de que se encontró que hasta un $10.4 \%$ de los hombres que la rechazaron mencionaron como principal motivo el temor a quedarse impotentes, podemos conside- rarlo como un porcentaje alto ya que estos varones habían tenido una consejería previa y habían sido ya referidos al centro estatal y a pesar de esto continuaron con creencias erróneas, reflejo de una falta de información adecuada. García-Moreno ${ }^{7}$ encontró que en el medio rural el temor a un mal desempeño sexual es la limitante más poderosa para rechazar la vasectomía, siendo por nuestros resultados similar al medio urbano y que es algo generalizado aún en otros países, como Nepal, Tanzania o India. ${ }^{17-19}$

Un resultado importante encontrado fue la importancia de quienes han recibido la información, cuando ésta fue otorgada por las amistades, existe una diferencia importante en el nivel de conocimientos entre los que aceptaron o rechazaron la vasectomía, que no se ve cuando la información es otorgada por otros medios incluyendo el personal de salud. Las amistades como una red social pueden influir en el uso de la anticoncepción en dos formas: al difundir información y al influir en el comportamiento. ${ }^{20}$ Los pacientes podrían darle un voto de confianza mayor a la experiencia directa o referencias secundarias de otras personas, que a la información otorgada por el personal de salud aquí entra el juego de la credibilidad, la comunicación, la empatía, que deben de darse en toda orientación. En México Echarri ${ }^{21}$ encontró que tanto hombres como mujeres reportan una falta de información sobre la salud sexual y reproductiva en fuentes oficiales así como la importancia de las redes sociales sobre el tema. Por otra parte Vargas ${ }^{22}$ reporta la importancia de las redes sociales para el inicio de la vida sexual, sin embargo no se encontraron estudios en México que reporten directamente la relación entre las redes sociales y el uso o elección de métodos de PF.

Nuestros resultados difieren a lo reportado por Untiveros ${ }^{5}$, donde resalta que la fuente de información más importante es el personal de salud, esto puede deberse 
a que en España, lugar donde se realizó el estudio, los programas de planificación familiar tiene un papel primordial en los servicios de salud, así como a otros factores socioculturales.

Esto refleja un área de oportunidad ya que la mejor promoción de la vasectomía podría venir de los propios hombres que se la han practicado. Cordoba-Basulto ${ }^{3}$ menciona que el reporte de experiencias de pacientes con vasectomía sin bisturí es un buen referente para tomar una decisión respecto a este método definitivo. Por otra parte, en la consultoría puede existir un flujo de información unilateral que no impacta en las decisiones de los pacientes. Mara Viveros ${ }^{23}$ reporta que la orientación sobre métodos de PF no permite que el usuario exprese sus verdaderas necesidades y temores, ligados o no al método que va a escoger. La respuesta de los orientadores se basa en plantear que los temores expresados no tienen fundamento científico, lo cual no ayuda mucho a resolver las inquietudes personales del usuario, quien a partir de ese momento se siente censu- rado para expresarse con libertad. El nivel de relación del orientador con el usuario es muy superficial, si se tiene en cuenta el tipo de preguntas que se le formula, el tiempo que se le destina y el carácter unívoco de la comunicación, desarrollado alrededor de un guión predeterminado.

Según los resultados de García-Moreno ${ }^{7}$ sus pacientes encuestados mencionaron que para que se tenga un mayor grado de aceptación de la vasectomía hay que cambiar principalmente las costumbres y tradiciones, la información sobre la operación y las ideas religiosas. Por otra parte es necesario tener personal de salud con conocimientos y actitudes favorables hacia el método. ${ }^{24}$

Dentro de las principales limitaciones que se presentaron, es que los resultados no reflejan certeramente la percepción de la población en general, solo pueden inferirse a los pacientes que acudieron a recibir una consejería y habían decidido hacerse la vasectomía y se refirieron al centro estatal, sin embargo se encontraron resultados similares a otros estudios.

\section{Conclusiones}

Podemos concluir que las amistades es la principal fuente de información de los pacientes, la paridad satisfecha es el principal motivo para aceptar la vasectomía y el desear tener mas hijos fue el principal motivo para rechazarla seguido del temor a la impotencia. Por otra parte encontramos que a mayor nivel escolar y mayor nivel de conocimientos sobre el método mayor aceptación del procedimiento. No existen diferencias significativas en la edad, edad de la pareja, número de hijos ocupación del paciente, ocupación de la pareja entre los varones que aceptaron y los que rechazaron la vasectomía. Se encontró que la red social de las amistades es importante para conocimientos apropiados sobre PF y la vasectomía, dando la pauta para aceptar o rechazar el método. Por otra parte cuando la información proviene de los prestadores de salud no existe diferencia en los conocimientos entre los varones que aceptaron o rechazaron el método. 


\section{Bibliografía}

1. Norma Oficial Mexicana, NOM 005-SSA2-1993, De los Servicios de Planificación Familiar. México: Secretaria de Salud: 2004

2. Vázquez-Jimenez AL, Suarez-Lugo N. Conocimiento y uso de métodos anticonceptivos en la población de 15 a 44 años del área urbana, Cunduacan. Tabasco 2005. Horizonte Sanitario 2006;5(2):8-25.

3. Córdoba-Basulto DI, . Valdepeña-Estrada R, PatiñoOsnaya SP Sapién-López JS, Rosas- Barrientos JV. Temores del varón que recurren a la vasectomía sin bisturí en el Centro de cirugía Ambulatoria de ISSSTE. Revista de Especialidades Médico-Quirúrgicas 2007;12(3):27-29

4. Díaz-Chávez EP, Mendez-Castorena $R$, MedinaChavez JL, Trujillo-Hernández B, Vásquez C. Vasectomía sin bisturí. Experiencia de 10 años. Rev Med IMSS 2004; 42(4):337-341.

5. Untiveros-Mayorga CF, Mauricio-Pachas J. Consecuencias a largo plazo de la vasectomía, en varones operados en el Hospital Nacional Cayetano Heredia. Departamento de Ginecología Obstetricia del Hospital Nacional Cayetano Heredia. Rev. Med. Hered 2004;15 (3):131-135

6. Cordoba-Basulto DI, Valdepeña-Estrada R, SapiénLópez JS, Salguero-Velázquez A, Patiño-Osnaya P. Recomendaciones sobre esterilización voluntaria que hacen los hombres con vasectomía sin bisturí. Revista de Especialidades Médico-Quirurgicas 2008; 13 (4):167172.

7. García-Moreno J, Solano-Saínos LM. Aceptación y rechazo de vasectomía en hombres del medio rural. Rev Med IMSS 2005;43(3):205-214.

8. Castro R, Díaz S, Gaán G, López C, Matarnala MI Normas Nacionales sobre Regulación de la Fertilidad. Chile: Ministerio de Salud; 2004.

9. Viveros-Vigoya M. La esterilización masculina: ¿un punto de inflexión en las trayectorias anticonceptivas y reproductivas? Reflexiones a partir de un estudio de caso colombiano. Sexualidad, Salud y Sociedad. Revista Latinoamericana. 2009 [Acceso 01 septiembre 2012], Disponible en http://www.e-publicacoes. uerj.br/index.php/SexualidadSaludySociedad/article/viewArticle/3/113

10. Quiroz Díaz NA, Gil-Henríquez A. Actitudes e información del varón relacionados a la aceptación de métodos anticonceptivos en zonas urbano- marginales de Trujillo". Revista Salud, Sexualidad y Sociedad 2009; 1(4): [Acceso 01 septiembre 2012], Disponible en: http://www.inppares.org/revistasss/Revista \% 20IV\% 202009/8-\% 20Varones\% 20MAC.pdf

11. Cordoba-Basulto DI, Ellos y la vasectomías: Temores, precauciones deseos y mitos de la sexualidad humana. Tesis doctoral. México ENAH,2005.

12. De la Cruz-Peñaran D, Langer-Glas A, Hernández-
Prado B, Gonzalez-Rengijo GF. Conocimiednto y actitudes de la pareja hacia la práctica de planificación famkiliar en la selva del Perú. Salud Pública Mex 2003:45(6):461-471.

13. Hernández-Onofre R. Creencias sobre el uso de anticonceptivos y masculinidad en jóvenes estudiantes del CETIS 42. D.F., México: Universidad Autónoma Metropolitana Unidad Iztapalapa; 2004.

14. Nian $C$, Xiaozhang $L$, Xiaofang $P$, Ping $Y$, Minxiang $L$. Factors influencing the declining trend of vasectomy in sichuan, China. Southeast Asian J Trop Med Public Health 2010;41(4):1008-1020.

15. Lara-Ricalde R, Velázquez-Ramirez N, Reyes-Muñoz E. Vasectomía sin bisturí. Perfil del usuario y resultados. Ginecol Obstet Mex 2010;78(4):226-231.

16. Pantelides E, Hernán M. Promoción de la Participación de los Hombres en la Salud Sexual y Reproductiva en Centroamérica. Análisis comparativo. 2005. [Acceso 01 septiembre 2012], Disponible en http:// www.cenep.org.ar/02_02_07.html

17. Bunce A. Guest $G$, Searing $H$, Frajzyngier V, Riwa $P$, Kanama J, Achwal I. Factors Affecting Vasectomy Acceptability in Tanzania. International Family Planning Perspectives 2007;33(1):13-21.

18. Mahat $K$, Pacheun $O$, Taechaboonsermsak P. Intention to Accept Vasectomy among Married Men in Kathmandu, Nepal. Asia Journal of Public Health 2010;1:8-14

19. Scott B, Alam D, Raman S. Factor affecting acceptance of vasectomy in Uttar Pradesh: Insights From community-based, participatory qualitative research. The RESPOND Project Study Series: Contributions to Global Knowledge- Report No. 3 New York: Engender Health/The RESPOND Project. 2011

20. Best K. Los contactos sociales influyen en el método. Network en español [serie en Internet]. 1999; [acceso citado 3 Ene 2013]; (19): [aprox. 10 p.] Disponible en: http://www.fhi360.org/sp/rh/pubs/ network/v19 4/social contacts.htm.

21. Echarri CJ. Barreras para la satisfacción de las necesidades de salud reproductiva: una experiencia desde la academia. El colegio de Médicos. [acceso citado 17 Oct 2013] Disponible en: http://www.alapop. org/2009/docs/echarri st3.pdf

22. Vargas-Valle ED, Martinez-Canizalez G, Potter JE. Religión e iniciación sexual premarital en México. Revista Latinoamérica de Población 2010;4 (7) 7-30.

23. Viveros $M$, Gomez F, Otero E. Las representaciones sociales sobre la esterilización masculina. El punto de vista de los orientadores del servicio de vasectomía en Clínica del Hombre, en Bogota, Colombia. Cad. Saúde Públ 1998;14:97-103.

24. Ebeigbe PN, Igberase GO, Eigbefoh J. Vasectomy: a survey of attitudes, counselling patterns and acceptance among nigerian resident gynaecologists. Ghana Medical Journal 2011; 45(3):101-104. 
ANEXOS

\section{UNIVERSIDAD AUTONOMA DE ZACATECAS \\ "FRANCISCO GARCIA SALINAS" \\ UNIDAD ACADEMICA DE ENFERMERÍA \\ CURSO COMPLEMENTARIO DE ENFERMERÍA}

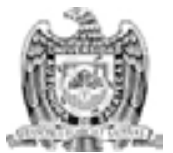

Somos estudiantes de Licenciatura en Enfermería de la UAZ, estamos realizando un protocolo de investigación acerca de los Factores socio-culturales para la aceptación o rechazo

de la vasectomía, por lo que nos es necesario obtener datos mediante esta cédula, cabe aclarar que la información que se recaude es confidencial y anónima y sólo será usada con fines de investigación científica.

INSTRUCCIONES. Subraye, tache o circule la respuesta que para ti sea la correcta.

PARTE I. DATOS GENERALES.

1. Edad:

2. Estado civil actual:

(1) Casado

(2) Soltero

(3) Unión libre

(4) Divorciado

3. Escolaridad:

(1) Analfabeta

(2) Primaria incompleta

(3) Primaria

(4) Secundaria
(5) Preparatoria
(6) Técnico
(7) Licenciatura
(8) Otros (especifique):

4. Municipio de procedencia:

(1) Aguascalientes
(2) Asientos
(3) Calvillo
(6) Jesús María
(7) Pabellón de Arteaga
(10) San José de Gracia
(4) Cosío
(8) Rincón de Romos

(5) El Llano

(9) San Fco. de los Romo

\section{Ocupación:}

(1) Empleado no Profesionista

(2) Obrero

(3) Comerciante

(4) Empleado Profesionista

(5) Trabaja por su cuenta

6. Religión que profesa:
(1) Católica
(2) Cristiana
(3) Testigo de Jehová

(4) Otra:

7. Número de hijos:
(1) 1
(2) 2
(3) 3
(4) 4
(5) 5 ó más

8. ¿De quién recibió información sobre la vasectomía?

(1) Familia (especifique quien):

(2) Amistades (especifique): 
(3) Personal de salud (especifique):

(4) Medios de información (especifique):

(5) Otros (especifique):

9. ¿Actualmente tiene pareja (esposa)? (NO TIENE, PASE A LA PREGUNTA 16)

(1) $\mathrm{SI}$

(2) NO

10. Años de matrimonio o de vivir juntos:

11. Edad de su pareja:

12. Escolaridad de su pareja:

(1) Analfabeta

(2) Primaria incompleta

(3) Primaria completa(7) Licenciatura

(4) Secundaria completa

(5) Preparatoria completa

(6) Técnico

13. Ocupación de su pareja:

(8) Otros (especifique):

(1) Hogar

(2) Empleada no Profesionista

(3) Comerciante

(4) Empleada Profesionista

(5) Trabaja por su cuenta

14. ¿Su pareja acepta la vasectomía?

(1) SI

(2) NO

¿Por qué?

15. ¿Su pareja lo acompaña a recibir información sobre la vasectomía?

(1) $\mathrm{S}$

(2) $\mathrm{NO}$

\section{PARTE II. CONOCIMIENTOS SOBRE VASECTOMÍA}

16. ¿Qué es un método anticonceptivo?

(1) Son aquellos en los se basa en el conocimiento de la fertilidad observando los síntomas asociados a la ovulación, sin el uso de fármacos ni procedimientos quirúrgicos.

(2) Son aquellos que se utilizan para limitar la capacidad reproductiva de un individuo o de una pareja, en forma temporal o permanente.

(3) Son aquellos que impiden el paso de los espermatozoides por la acción de un obstáculo físico.

(4) Es aquel en que la mujer cuenta los días de su ciclo menstrual para determinar los días que esté más fértil.

17. Los siguientes son métodos anticonceptivos:\}

(1) Condón femenino, anillo vaginal, implante subdérmico, salpingoclasía.

(2) Dispositivo (DIU), pastilla de emergencia, hormonas orales.

(3) Coito interrumpido, vasectomía, métodos naturales (Billings, Ritmo)

(4) Todas las anteriores.

18. Los siguientes son métodos anticonceptivos permanentes o definitivos:

(1) Salpingoclasía

(2) Dispositivo (DIU)

(3) Vasectomía

(4) Sólo (1) y (3)\}

19. ¿Qué es la vasectomía?

(1) Es un método anticonceptivo permanente para la mujer, que consiste en la obstrucción bilateral de las trompas uterinas.

(2) Es una operación quirúrgica para quitar la próstata.

(3) Es la incapacidad del individuo (mujer u hombre) para reproducir un hijo vivo.

(4) Es un método anticonceptivo permanente para el hombre, que consiste en la sección

o ligadura bilateral de los conductos deferentes, con el fin de evitar el paso de los espermatozoides. 
20. ¿Cuál es la forma de hacer la vasectomía?

(1) En quirófano (técnica quirúrgica habitual)

(2) En casa

(3) En un consultorio (vasectomía sin bisturí)

(4) Sólo (1) y (3)

21. Después de la vasectomía, ¿cuánto tiempo deberá esperar para el primer análisis de semen?

(1) A la semana

(2) Al mes

(3) $\mathrm{Al}$ año

(4) De 2 a 3 meses (aprox. 20 a 25 eyaculaciones)

22. Si en el primer análisis de semen aún hay espermatozoides, ¿cada cuánto tiempo deberá hacerse el análisis? (1) Diario

(2) Cada semana

(3) Cada mes, hasta que el resultado de ausencia de espermatozoides total

(4) Nunca

23. ¿Durante cuánto tiempo deberá seguir utilizando otro método anticonceptivo, después de la vasectomía?

(1) Siempre

(2) Una semana

(3) Un año

(4) Hasta que el análisis de semen de como resultado ausencia total de espermatozoides

24. Después de la vasectomía, ¿cuánto tiempo debería esperar para volver a tener relaciones sexuales?

(1) 24 horas (1 día)

(2) 72 horas (3 días)

(3) Una semana

(4) Un mes

25. Después de la vasectomía, ¿qué actividades no debería realizar?

(1) Ningún tipo de actividad

(2) Puedo hacer todo tipo de actividad

(3) Ejercicio físico violento o deportes con riesgo no controlado

(4) Ninguna de las anteriores

26. ¿Por cuánto tiempo no deberá realizar las actividades antes mencionadas?

(1) Un día

(2) Una semana

(3) Un mes

(4) Un año

\section{PARTE III. INFORMACIÓN SOBRE VASECTOMÍA}

27. Mencione el o los motivos por el cual aceptó o rechazó la vasectomía: (forzar la respuesta exhaustiva al agregar al final, preguntas como: ¿Qué más me puede decir al respecto?, ¿Algo mas que quiera decirme de esto?)

28. ¿Cree usted que con la vasectomía quedará impotente?

(1) $\mathrm{Si}$

(2) No

29. ¿Cree usted que la vasectomía afecta la calidad de la erección, la eyaculación y el orgasmo?

(1) $\mathrm{Si}$

(2) No

30. ¿Cree usted que la vasectomía tiene efectos secundarios?

$\begin{array}{ll}\text { (1) } \mathrm{Si} & \text { (2) No ¿Cuáles? }\end{array}$

31. ¿Sabe usted en qué lugar hacen las vasectomías, aquí en Aguascalientes?

(1) $\mathrm{Si}$

(2) No Mencione el lugar

32. ¿Sabe usted quién realiza la vasectomía? 


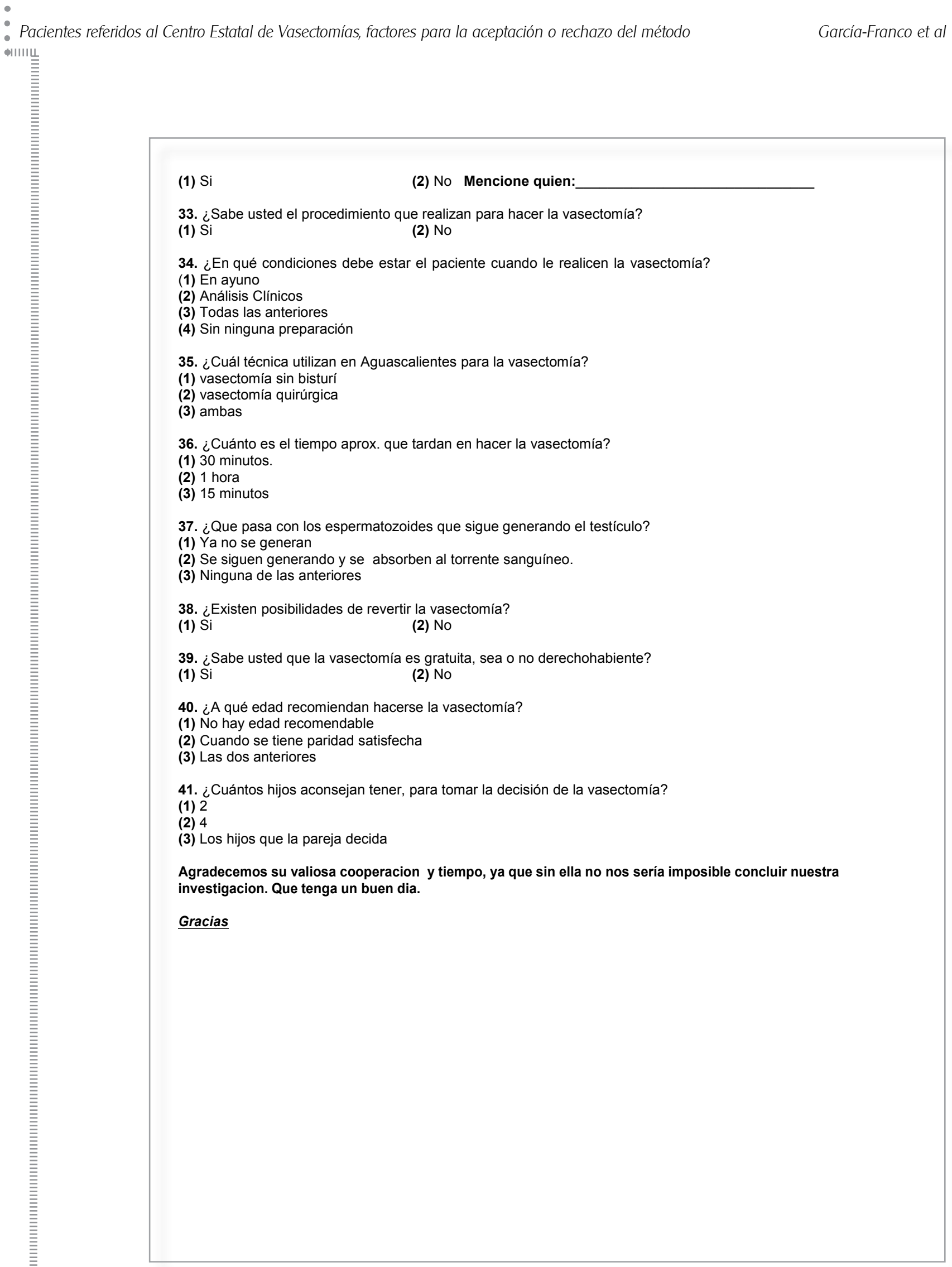

